\title{
An Expert System-Driven Method for Parametric Trajectory Optimization During Conceptual Design
}

\author{
Patrick D. Dees* and Mathew R. Zwack ${ }^{\dagger}$ \\ Jacobs ESSSA Group, Huntsville, AL, 35806, United States \\ Michael Steffens ${ }^{\ddagger}$, Stephen Edwards ${ }^{\S}$, Manuel J. Diaz \\ Georgia Institute of Technology, Aerospace Systems Design Laboratory, Atlanta, GA, 30312, United States \\ James B. Holt"l \\ NASA, George C. Marshall Space Flight Center, AL, 35812, United States
}

\begin{abstract}
During the early phases of engineering design, the costs committed are high, costs incurred are low, and the design freedom is high. It is well documented that decisions made in these early design phases drive the entire design's life cycle cost. In a traditional paradigm, key design decisions are made when little is known about the design. As the design matures, design changes become more difficult in both cost and schedule to enact. The current capability-based paradigm, which has emerged because of the constrained economic environment, calls for the infusion of knowledge usually acquired during later design phases into earlier design phases, i.e. bringing knowledge acquired during preliminary and detailed design into pre-conceptual and conceptual design. An area of critical importance to launch vehicle design is the optimization of its ascent trajectory, as the optimal trajectory will be able to take full advantage of the launch vehicle's capability to deliver a maximum amount of payload into orbit. Hence, the optimal ascent trajectory plays an important role in the vehicle's affordability posture yet little of the information required to successfully optimize a trajectory is known early in the design phase. Thus, the current paradigm of optimizing ascent trajectories involves generating point solutions for every change in a vehicle's design parameters. This is often a very tedious, manual, and time-consuming task for the analysts. Moreover, the trajectory design space is highly nonlinear and multi-modal due to the interaction of various constraints. When these obstacles are coupled with the Program to Optimize Simulated Trajectories (POST), an industry standard program to optimize ascent trajectories that is difficult to use, expert trajectory analysts are required to effectively optimize a vehicle's ascent trajectory. Over the course of this paper, the authors discuss a methodology developed at NASA Marshall's Advanced Concepts Office to address these issues. The methodology is two-fold: first, capture the heuristics developed by human analysts over their many years of experience; and secondly, leverage the power of modern computing to evaluate multiple trajectories simultaneously and therefore enable the exploration of the trajectory's design space early during the preconceptual and conceptual phases of design. This methodology is coupled with design of experiments in order to train surrogate models, which enables trajectory design space visualization and parametric optimal ascent trajectory information to be available when early design decisions are being made.
\end{abstract}

${ }^{*}$ Trajectory Engineer, Advanced Concepts Office, George C. Marshall Space Flight Center/ED04, Huntsville, AL, AIAA Member.

†Systems Engineer, Advanced Concepts Office, George C. Marshall Space Flight Center/ED04, Huntsville, AL, AIAA Member.

${ }^{\ddagger}$ PhD Candidate, School of Aerospace Engineering, 270 Ferst Drive, Mail Stop 0150, AIAA Student Member.

$\S$ Research Engineer, School of Aerospace Engineering, 270 Ferst Drive, Mail Stop 0150, AIAA Member.

『Graduate Student, School of Aerospace Engineering, 270 Ferst Drive, Mail Stop 0150, AIAA Student Member.

" Lead Aerospace Engineer, Advanced Concepts Office, George C. Marshall Space Flight Center/ED04, Huntsville, AL 


\section{Nomenclature}

DOE Design of Experiments

POST Program to Optimize Simulated Trajectories

RSE Response Surface Equation

$S L S \quad$ Space Launch System

$V \& V \quad$ Verification and Validation

\section{Introduction}

Launch vehicles are complex systems used to transport expensive, often one-of-a-kind payloads to earth orbit and beyond. Due to the challenges associated with earth to orbit launch, these vehicles frequently require billions of dollars to develop. ${ }^{1}$ Large development costs then translate to high prices to launch a given payload, which can range from $\$ 50 \mathrm{M}-\$ 500 \mathrm{M}$ per launch or $\$ 20 \mathrm{k}-\$ 30 \mathrm{k}$ per kilogram. ${ }^{1,2}$ In today's increasingly budget-constrained environment, affordability is tantamount to the successful progression of a new launch vehicle program.

The Department of Defense Acquisition Guidebook defines affordability as the "degree to which the capability benefits are worth the system's total life-cycle cost". ${ }^{3}$ In this sense, an affordable vehicle is one that provides sufficient capability to meet the program requirements at a cost that will fit within budget and schedule constraints. Improvement in affordability can thus be achieved by finding a balance between maximum capability and minimum cost.

During early conceptual design, decisions are made to begin identification of a baseline vehicle. With the selection of a baseline concept the maximum expected performance of the vehicle will essentially be locked-in. Other vehicle metrics such as reliability, safety, manufacturability, and operations cost will also be impacted by concept down selection. ${ }^{4-7}$ Due to the broad effect of these early design decisions, upwards of $80 \%$ of the vehicle's life-cycle cost is committed during early design. ${ }^{4}$ Since affordability is a function of life-cycle cost it will be profoundly impacted by the decisions made during conceptual design.

To improve vehicle affordability it is important that concept down selection be made using more design knowledge. Improvement in design knowledge can be achieved in multiple ways including the use of higher fidelity tools, a broader suite of disciplinary tools, and exploration of a large number of concepts. As an example, for launch vehicles, exploration of large trade spaces can be achieved using the rocket equation for performance estimation. ${ }^{6}$ This analysis simplifies the trajectory and structural analysis into a mass ratio and gravity constant. Thousands of vehicle concepts can be evaluated in this manner, but an accurate picture of the expected vehicle performance requires the use of higher fidelity trajectory tools to accurately capture ascent losses.

During conceptual design the trajectory analysis becomes a bottle-neck for all the other disciplines. This is due in part to the fact that the trajectory is heavily dependent upon these other disciplines. It is also due to the complex nature of trajectory optimization tools, which more accurately capture ascent losses. Such trajectory tools do not offer a solution in a closed form like the rocket equation. Instead they require the solution of an optimal control problem along the entire ascent trajectory, which can be very time consuming for large trade spaces. For this reason, a middle ground is sought between the closed form rocket equation and more accurate but time consuming trajectory optimization tools. The desired approach will provide a closed form solution in order to enable rapid evaluation of large trade spaces, while accurately accounting for ascent losses.

The concept of surrogate modeling was identified as a candidate for representation of the trajectory discipline. A surrogate model is a mathematical approximation of a set of data, usually from a computationally expensive analysis code. ${ }^{8}$ It utilizes a closed form equation to provide rapid estimates of the output of the detailed analysis. Creation of a surrogate model for a trajectory optimization program will allow for the exploration of large design spaces, as done with the rocket equation, while simultaneously capturing ascent losses. 


\section{Background}

Prior to generating a surrogate model for the trajectory discipline the tool of interest must be identified. For launch vehicle conceptual design, trajectory optimization is typically performed using one of two tools: Program to Optimize Simulated Trajectories (POST) and Optimal Trajectories By Implicit Simulation (OTIS). These tools are both widely accepted by the launch vehicle design community and utilize direct shooting and direct collocation optimization schemes, respectively. ${ }^{9}$ The POST tool will be used in this paper because it is currently applied within the Advanced Concepts Office at Marshall Space Flight Center to evaluate conceptual launch vehicles. ${ }^{10}$

In order to generate a surrogate model, data from POST is required. The amount of data required for the model depends heavily upon the number of input variables of interest and the desired accuracy of the model. Application of the model across a large trade space will require a data set that captures the behavior of POST within the space. Since the trajectory space is expected to be highly multi-modal, it is expected that on the order of 1,000 points will be required to generate a surrogate model with an acceptable level of accuracy.

The primary challenge for creating a surrogate model lies in the generation of the initial data set. This challenge is due to the difficulty associated with closing cases in POST. For an experienced POST analyst it may be possible to close up to 5 unique vehicles in one day. At this rate, generating enough data for a POST surrogate would take many months. Therefore, the goal of this work is to develop an approach for automating the closure of unique vehicle cases in POST. This will ultimately enable creation of a POST surrogate for use during a conceptual design study.

To begin the development of a POST automation approach, requirements for the method will be derived. As noted above, closing cases by hand in POST can prove to be difficult due to failed trajectories and program crashes. Therefore the first requirement states that the automation method must be able to handle failed trajectories and POST crashes without requiring an expert in the loop.

The second requirement states that the method must be able to run a broad range of vehicle concepts. This requirement stems from the desire to generate a surrogate model that can be used for design space exploration. In order to maximize the utility of the surrogate in this application the data set must capture the behavior of POST for a variety of input variables and ranges.

The third requirement also stems from the desire to use the surrogate model during conceptual design. Typical conceptual design studies are carried out in a short time frame; on the order of weeks. ${ }^{11}$ Since a POST surrogate model may require thousands of points to achieve an acceptable fit the automation method must be able to close cases in a rapid manner. Generically stated, the method must be able to provide the data set in a time frame that is reasonable for a conceptual design study.

The previous requirements call for a method that can produce many POST cases without including an expert in the loop. This implies that the automation will produce closed cases in the same manner as an expert. However, due to the multi-modal nature of trajectory optimization the expert and automated solutions are not guaranteed to match for each vehicle. Therefore a final requirement must be added, which states that the method must be transparent and provide an easy means for validation and verification. Completing Verification and Validation $(\mathrm{V} \& \mathrm{~V})$ on the automated approach is a vital step towards producing data that will ultimately be used for concept down selection. The final list of method requirements are itemized below.

- Method must handle failed trajectories and POST crashes

- Method must be able to run a broad range of vehicle concepts

- Method must generate data set in a timeframe that is reasonable for a conceptual design study

- Method must be transparent and provide easy means for validation and verification 


\section{Approach}

In the following section, the requirements previously placed upon the work are explored. At each step, another piece of the final tool is incorporated and justified, and any pitfalls associated with their inclusion presented. In the end their synthesis results in the creation of a tool called multiPOST, whose parts are discussed in further detail in the following section.

\section{III.A. Sampling}

In order to create a surrogate model of POST which can represent any class of vehicle within a trade study's bounds, the targeted design space must be thoroughly sampled. In order to do so in a quick turnaround timeframe (on the order of one month), the sampling must be executed as efficiently as possible. Design of Experiments (DOE) is thus utilized to maximize the information gained on the outputs while simultaneously minimizing effort (i.e. cases run) by mathematically structuring the selection of inputs. There are many different DOE schemes to choose from, and the algorithm by which points are calculated will vary from one scheme to the next. While the vehicle design space is most easily expressed in a Cartesian system of variables with upper and lower bounds, the majority of the reasonable designs are expected to come from the interior of the design space rather than from the edges or corners. In addition, there is a desire to avoid the corners of this Cartesian space, as trajectory optimization tools like POST tend to have difficulty finding feasible trajectories for extreme combinations of vehicle designs. These considerations lead naturally to the selection of a space-filling DOE.

Among space-filling DOEs, there are a variety of approaches to sampling, including such strategies as Sphere-Packing, Latin Hypercube, and Maximum Entropy designs, among others. ${ }^{12}$ Each strategy is based on a different mathematical formulation or figure of merit, so depending on the particular application, certain strategies may be more appropriate than others. As mentioned previously, getting certain trajectories to converge in POST can sometimes be difficult to the point where it may be desirable to move on and mark a particular case as failed. This is an undesirable outcome in DOEs based on the optimization of some statistical metric, as failing to gather data at the selected points represents a compromise of the original sample design. If too many cases fail this can become problematic, as the failures may introduce appreciable correlation in the sample. However, Uniform DOEs do not suffer as quickly from failed cases, as removing points at random does not compromise the statistical nature of the design. For this reason, a Uniform DOE was used in this work.

For the trajectory discipline, there are two levels of inputs required to fly the vehicle. The first, vehiclelevel parameters, is the collection of masses, thrusts, etc. which describes the vehicle on the pad. As the desired surrogate model will be a function of these physical parameters, the DOE scheme chosen above is to be used for selecting this level of inputs. The second, control-level parameters, is the collection of pitch rates, launch conditions, etc. which describes how the vehicle flies. These control-level parameters are not explicitly known, and while hypothetically one would expect there to be a relationship between the vehiclelevel parameters and the optimal set of control-level parameters, previous efforts to elicit a mapping between the two have met with difficulty. ${ }^{13}$ Combining knowledge of previous difficulties with the expectation that output response to control-level inputs is nonlinear, a complex relationship between vehicle-level and controllevel inputs is anticipated. Motivated by this expected correlative complexity, sampling of the vehicle-level and control-level inputs have been separated into a two-level problem.

The sampling approach applied to the control-level parameter space is based on an understanding of the characteristics and behaviors of POST. Due to the difficulties that arise occasionally in getting POST to converge, there is often a particular sensitivity to the initial control parameters provided as an initial guess. If the feasible region in the control-level parameter space for a desired orbit and particular selection of vehicle-level parameters is small, then a single set of control-level inputs (DOE set) can completely miss the feasible region, giving the false impression that a vehicle cannot fly the mission specified. Also, since POST employs a gradient-based (local) optimizer, ${ }^{14}$ it has the drawback of finding only local minima; this poses a problem, as previous efforts ${ }^{15,16}$ have shown that the trajectory output space is multimodal. It is undesirable to get stuck using suboptimal data, even though POST may have returned it as optimized.

Thus, with no explicit a priori knowledge of the control parameters, the expectation that the corresponding output space will be nonlinear and multimodal, and the knowledge that the optimizer in use is local, some method to enable a broader, if not global, search is needed. A statistically rigorous process is applied to meet this need, which will be published in a separate paper at a later date; however, the essence of the 
approach proceeds as follows: A minimum to maximum range is identified for each parameter in the controls, loosely leveraging expected values and correlations set forward by a subject matter expert; and random cases are selected and evaluated from these ranges until specific statistical thresholds are achieved. The response of interest is then extracted from the resulting data set.

\section{III.B. Heuristics}

For a given vehicle attempting to achieve a given orbit, flying with randomly selected controls can be quite disastrous. POST experts apply heuristics to the trajectory inputs between runs to move arbitrarily chosen controls to a locally optimal neighborhood so that POSTs internal optimizer can take over. These heuristics were applied in the tool and verification was conducted via interviews with current analysts in ACO to fine-tune operations. In this manner, the developed tool can be thought of as simply an automated version of the current method. Historically a human analyst has used their best guess at a vehicle's flight path as their initial guess, then used their best judgment to nudge the path this way or that in order to produce an acceptable trajectory. This issue is a consequence of the fact that POST employs a direct shooting method. ${ }^{14}$ Examples of these heuristics are pitching up if a vehicle goes below a certain altitude; pitching down if the maximum altitude is violated; and adjust pitch events in opposing directions if there is a negative mass error. These adjustments are achieved by multiplying the control-level parameter inputs by user-specified constants. Feasible trajectories (i.e. trajectories which achieve the desired orbital parameters within acceptable tolerances) that are satisfactorily optimized are recorded and then re-optimized. This process is repeated until an absolute tolerance on the difference between iteration results is satisfied, at which point the trajectory is recorded as converged.

Many of the difficulties of working with POST in particular can be summed up by the statement that POST is only as good as the algorithms it contains. It is an optimizer, not a pilot. An example of this behavior can be seen in Figure 1. The chart represents the collection of over 30 feasible trajectories, plotted on the left as altitude versus time, and on the right as dynamic pressure versus time. The lighter blue trajectories are less desirable (based on the final mass injected into orbit) while the lighter purple are more desirable. The optimizer in POST led to an optimal trajectory that was actually infeasible in reality. In this case the manner in which the optimizer achieved a higher optimized variable was to dip back into the atmosphere, subjecting the vehicle to intense aeroheating. To POST this is the peak of efficiency, but this trajectory will never be physically flown, and reporting metrics from this trajectory would lead to inaccurate conclusions. In order to restrict this behavior, these trajectories are removed by filtering the data after it is generated. In this case the dynamic pressure is limited to a specified value after the gravity turn has completed, and to a lower specified value after upper stage ignition. Violating the former of these constraints is a good indicator that the vehicle has dipped back into the atmosphere, and violating the latter of these constraints could constitute thermal damage to the payload. In addition, the time history of the altitude is examined to ensure that if the vehicle reaches a local maximum, no more than $1000 \mathrm{ft}$ of altitude is lost before reaching a local minimum. Another way POST can lead to inaccurate results is when the optimized variable value is traded for a better orbit. In the case where the optimized variable is the payload, which normally is desired maximized, POST will sometimes decrease this value in order to get the vehicle to the desired orbit. This behavior has been found primarily in situations where the combination of vehicle and steering-level parameters is poor, such as a vehicle with a low thrust-to-weight flying a sharp gravity turn. In order to exclude this behavior the history of runs for a particular repetition is tracked, and if the payload decreases twice in a row, or decreases by a large amount the trajectory is considered unacceptable and discarded. 

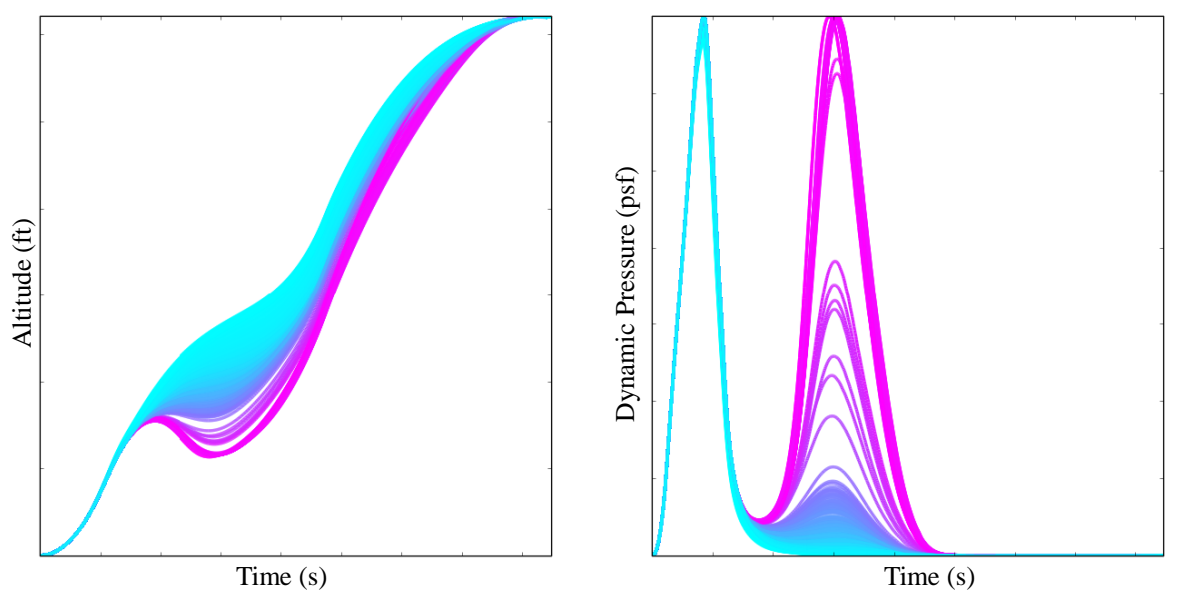

Figure 1: March of POST

In addition there are some POST-specific errors for which any automated tool must be able to handle. The first case is when POST simply gets stuck somewhere in its calculations. The user would simply see no more output coming back from the program over command line and after waiting a certain amount of time would simply end execution. Another similar error is the 'georate' error, which is caused by an error in a specific POST-internal calculation. The user would simply see that word show up in command line and nothing more coming after, and so would end execution. Finally, a 'Run-time Error' can occur when a vehicle stage achieves a negative mass during a phase. When transitioning from one phase to another, the negative mass will cause a logarithmic domain error and end the run. In this case, the user would see this as analogous to a trajectory ending with 'Negative Mass' in the Failed Case Summary and adjust the control parameters accordingly. For each of these error cases, the heuristics are designed to act in exactly the same manner as a human analyst.

\section{III.C. Automation}

The set of cases within the vehicle-level parameters chosen for analysis by DOE, coupled with the arbitrarily large random selection of points within the control-level parameters represents a large amount of information gathering. The philosophy employed for automation of trajectory analysis has been to mimic the manual process by which a human POST expert will evaluate a given vehicle. In this manner the current state-of-theart in terms of discipline analysis knowledge is preserved, while now leveraging computer time over human time. However, a POST expert is still required in the design loop for the verification and validation of both inputs and outputs of the tool. Since the tool is simply a set of scripts running an existing tool, verification was performed by running test cases via arbitrary selection of vehicle and control-level parameters both through the tool and manually. The heuristics, which were derived from interviews with POST analysts including the first author, were verified in the same manner as the overall execution of the tool, by manually stepping through the same process as the tool. Manually running POST requires the POST executable, a properly formatted text input file, and any external files required. These external files can include atmosphere and winds models, thrust traces for SRBs, etc. The POST executable will only read in a file named s.inp and any specified include files within its current directory, and outputs files in its current directory. Due to this limitation, parallel runs of the program require separate directories housing copies of the executable and any required files. Motivated by this capability for parallelization, the tool is designed to take advantage of parallel processing. Each parallel run of POST requires some agent to shepherd it, analyze the output, make any adjustments to the inputs necessary, and repeat the process as necessary.

\section{IV. multiPOST}

This section will give an overview of the tool, named multiPOST, which deploys multiple agents approximating human analysts. Although there have been previous efforts to apply multiprocessing to POST, ${ }^{17}$ this effort focuses on parallelizing the entire program instead of internal algorithms. This parallelization 
takes the form of executing many instances of POST. The tool was thus designed to have automated agents named ChildPOST working in separate directories with separate copies of the POST executable and orchestrating the runs of each repetition as heuristics dictate, receiving repetitions from a central agent named MotherPOST which also handles parsing the results of successful trajectories. Some detail on these codes will be presented next. In addition to the codes, an Excel document named POSTInputs is used to store the DOE cases and specific MotherPOST execution flags which shape how the user wishes to use the tool, and a text input file (which must be named s.inp) which can be altered to represent different vehicles within the trade study bounds. An overview of the tool can be seen in Figure 2 below.

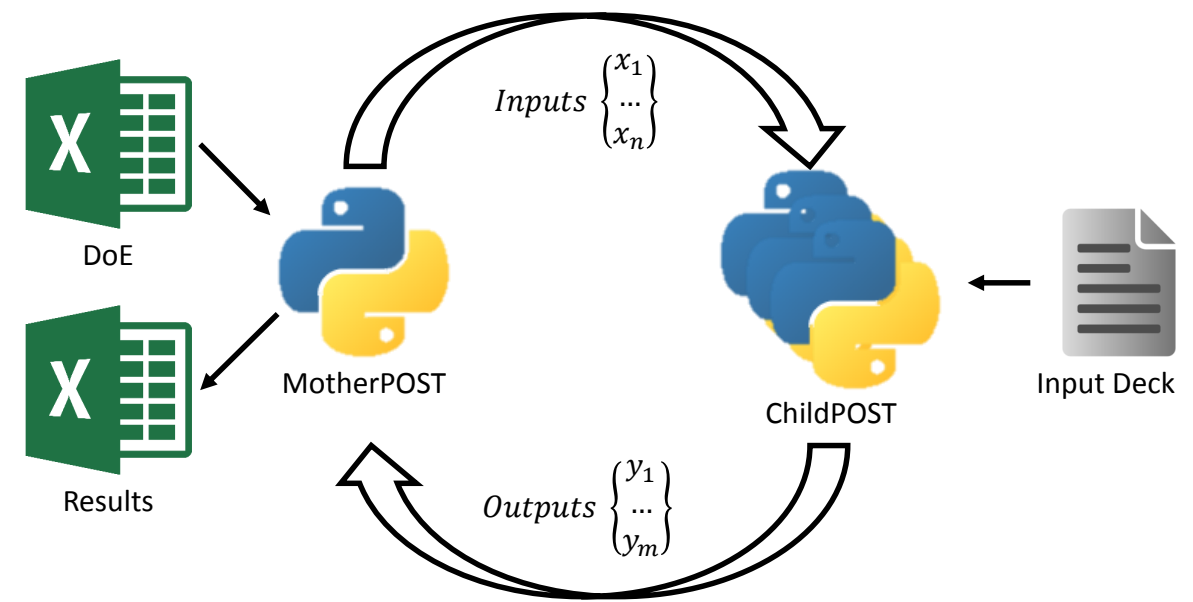

Figure 2: multiPOST Overview

\section{IV.A. POSTInputs}

The only portion of the tool the user must alter is an Excel file called POSTInputs, which houses all the important inputs and output definitions. One sheet contains the DOE information on vehicle-level parameters, another contains a table of output variables to retrieve from each vehicle trajectory, and another contains inputs such as the ranges through which to randomly select control-level parameters, what kind of vehicle to fly, and how many times a vehicle configuration must achieve a converged orbit before the case is considered done.

\section{IV.B. MotherPOST}

One of the two main codes of the program, MotherPOST, is responsible for reading in all the information contained in the POSTInputs document. The inputs contained therein define how the program is to execute analysis. MotherPOST then creates a certain number of ChildPOST child processes to handle individual runs of POST. For each case of the DOE, MotherPOST creates randomly selected repetitions, which are the control-level parameters. These are joined with the vehicle-level parameters and fed into a queue of runs from which the child processes pull jobs. As results come back from ChildPOST processes, the MotherPOST process is responsible for parsing output files and converting them into a comma-separated values table.

\section{IV.C. ChildPOST}

An instance of ChildPOST pulls from the queue created by MotherPOST and performs the repetition by first creating a POST input deck with the gathered inputs, activating the POST executable, and finally partially parsing the output data. The heuristics then determine the next step. A ChildPOST instance will iterate on a particular repetition until either convergence criteria are satisfied, or the repetition is flagged as unusable. If over a few runs a repetition achieves convergence, its identifying data is sent back to MotherPOST, and the output file moved to a results folder for further parsing. An overview of this code can be found in Figure 3 below. 


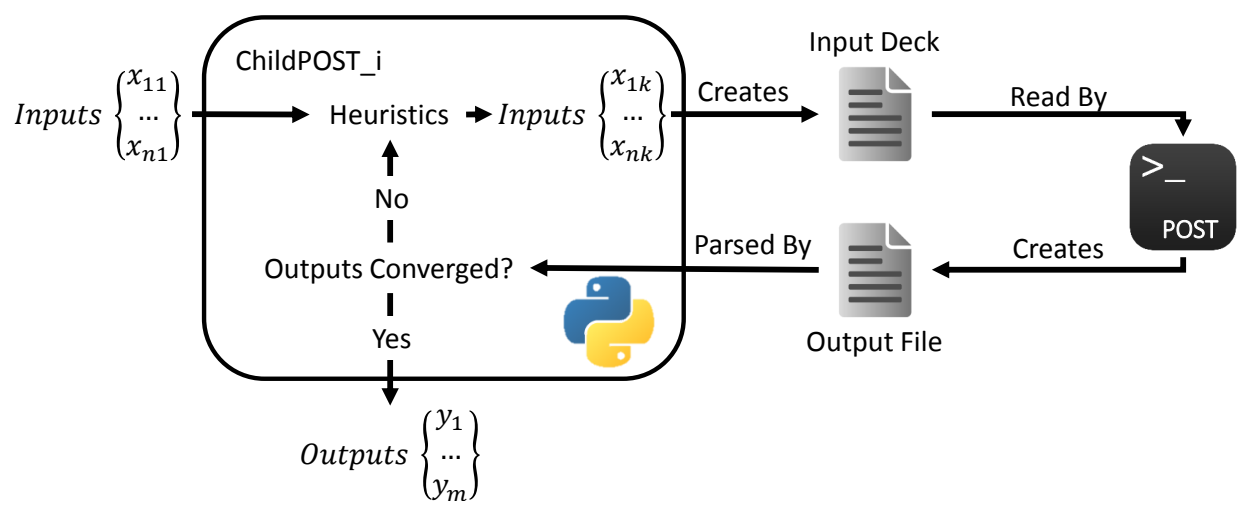

Figure 3: ChildPOST Detail

\section{Example Problem}

The example problem is based on the Space Launch System (SLS). The SLS is currently being developed by NASA as the next generation heavy lift launch vehicle, which will enable manned exploration missions to the moon and beyond. ${ }^{18}$ The SLS was chosen as a relevant example because design trade studies are currently being performed for future block upgrades of the vehicle. These upgrades will ultimately affect the vehicle's boosters and upper stage. Changes to these elements represent a wide variety of architecture options that will have an effect on the vehicle's trajectory. Thus, the SLS was deemed to be a good example vehicle for application of the method.

The SLS is a 2.5 stage vehicle with liquid hydrogen and liquid oxygen core and upper stages. ${ }^{18}$ The baseline SLS vehicle utilizes two STS derived five-segment solid rocket boosters, but advanced solid and liquid boosters have been proposed for future versions. In order to narrow the scope of the example problem no modifications will be made to the solid rocket boosters. In addition, the core stage of the vehicle will be fixed to the baseline configuration with 4 RS-25 engines. Therefore, the example problem will focus on capturing design trades for the upper stage of an SLS-like vehicle.

Trades of interest for the upper stage include number of engines, thrust per engine, specific impulse, engine mass, engine dimensions, and component materials. The POST input deck was parameterized to capture the effects of these parameters on the payload delivered to orbit. In order to minimize the required number of DOE cases, the number of input variables was reduced. Due to dependencies between the variables it was possible to reduce the number of input parameters while maintaining the capture of the desired trades.

First, the mass of the upper stage was represented as a gross mass. This variable is dependent upon many other parameters such as number of engines, engine mass, and component materials. Note that the gross mass of the upper stage also includes the payload being delivered to orbit. Although the gross mass effectively absorbs multiple variables, trades such as component materials and engine mass can still be incorporated using the appropriate mass differences.

Instead of representing the total thrust of the upper stage as number of engines times the thrust per engine, a total thrust to weight was used. This was done to remove a discrete numeric variable, number of engines, from the list of DOE inputs. Including discrete inputs within a DOE will rapidly increase the number of points required to achieve an acceptable surrogate model fit. The range for total thrust to weight of the upper stage was set up to represent a three to five engine configuration with thrust per engine between 20,000 and 40,000 pounds.

The next two input variables, $I_{s p}$ and total exit area, represent the upper stage engine selection. The $I_{s p}$ range was created based upon reasonable levels for a liquid hydrogen, liquid oxygen upper stage engine. The values for total exit area are related to the exit diameter of the engine as well as the number of engines on the stage. The range for this variable was set up to capture three to five engines with exit diameters similar to existing upper stage engines.

As noted above, the core of the vehicle will be fixed in terms of number of engines, thrust per engine, and propellant loading. However, a variable upper stage mass will have an effect on the structural design of the core vehicle. A heavier upper stage will ultimately require a bulkier core structure to support it on the pad and early in the trajectory. Therefore, core burnout mass was included in the list of DOE inputs. The range for core burnout was developed using structural mass estimates for the lightest and heaviest upper 
stage configurations.

The last two DOE input variables for the example problem are structural constraints on the trajectory. The max $\mathrm{G}$ variable limits the acceleration during the ascent, while max $\mathrm{Q}$ limits the aerodynamic loading on the vehicle. The ranges for max $\mathrm{G}$ and $\max \mathrm{Q}$ were derived from prior experience with running SLS-like vehicles within POST.

The final set of DOE inputs and ranges can be seen in Table 1. In addition to these variables, multiple other parameters were fixed while carrying out the example problem. Table 2 gives a list of fixed values used during the DOE runs. The core engine parameters were derived from RS-25 specifications published by Aeroject Rocketdyne. ${ }^{19}$ The booster parameters in Table 2 were taken from the 5 segment reusable solid rocket motor shown in the ATK space propulsion products catalog. ${ }^{20}$

Table 1: POST DOE Inputs

\begin{tabular}{|c|c|}
\hline Variable & Range \\
\hline Upper stage gross mass & $340,000-640,000 \mathrm{lbs}$ \\
\hline Upper stage T/W & $0.2-0.75$ \\
\hline Upper stage Isp & $440-470 \mathrm{sec}$ \\
\hline Upper stage total exit area & $35-235 \mathrm{ft}^{2}$ \\
\hline Core burnout mass & $240,000-308,000 \mathrm{lbs}$ \\
\hline Max G & $3-4$ \\
\hline Max Q & $540-700 \mathrm{psf}$ \\
\hline
\end{tabular}

Table 2: POST Fixed Parameters

\begin{tabular}{|c|c|}
\hline Parameter & Value \\
\hline Core number of engines & 4 \\
\hline Core engine sea level Isp & $366 \mathrm{sec}$ \\
\hline Core sea level thrust per engine & $418,000 \mathrm{lbf}$ \\
\hline Core propellant mass & SLS Baseline \\
\hline Booster burnout mass & $181,000 \mathrm{lbs}$ \\
\hline Booster propellant mass & $2,800,000 \mathrm{lbs}$ \\
\hline Payload fairing type & $90 \mathrm{ft} \mathrm{w} / \mathrm{ogive}$ nose \\
\hline Payload fairing mass & $20,000 \mathrm{lbs}$ \\
\hline
\end{tabular}

After identifying the inputs and ranges of interest a DOE can be set up. As discussed in Section III.A, many options exist for generating a DOE including space filling, factorial, and composite designs. A Uniform space filling design was ultimately selected for use in the example problem.

Due to known issues with closing cases in POST a large uniform DOE of 2,500 design points was used. This ensured that a sufficient sampling of the design space was completed despite a very low pass rate. For each of the 2,500 design points 25 repetitions were run using multiPOST. These repetitions represent randomly generated initial control parameter guesses. The number of repetitions was selected to balance the required runtime of the DOE and the number of successful cases. Increasing the repetitions increases the probability of returning a closed trajectory for each DOE point, however, it also greatly increases the runtime. To complete this DOE a total of 62,500 unique POST runs were required. 


\section{Results}

The uniform DOE runs were split over two workstations, each with 24 cores. Approximately 48 hours of runtime was required to complete the DOE. Out of 62,500 unique repetitions, around 42,000 were successful. However, as discussed in Section III.B, not all of these trajectories can be realistically flown by the vehicle. Therefore, filters were applied to the raw data set to remove unrealistic trajectories.

Following the filtering, 31,000 successful repetitions remained, which were then grouped by case number. Out of the original 2,500 DOE points, 1,644 had at least 1 successful repetition. For cases with multiple successful repetitions the maximum injected mass was taken as the output for the vehicle. The maximum was taken to more closely replicate the results from an analyst running POST by hand. Many of the successful trajectories that fell below the maximum injected mass for that vehicle could easily be adjusted by an expert to achieve a more optimal value. Therefore, they were ignored for surrogate model fitting purposes.

The surrogate model fitting was performed using the statistical software JMP. This software provides tools for fitting many types of surrogates including neural networks, Gaussian process models, and response surface equations. A response surface equation (RSE) was selected as the desired fit for the example problem data. An RSE was selected primarily because of its compact form in comparison to a neural net or Gaussian process model.

Multiple different options are provided in JMP for fitting response surface equations. Because of the nonlinear nature of the data, a stepwise regression was used to fit the RSE. Stepwise regression within JMP uses a statistical significance level to determine which terms in the regression model are most beneficial to the model's ability to predict the data. At each step during the model fitting, the significance levels are recalculated and terms are added or removed. This operation is performed until a pre-selected stopping criterion is met. Using this approach allows for the use of only the significant terms within the model.

Following the stepwise regression, multiple goodness of fit tests must be applied to ensure the RSE is accurate enough for use during a conceptual design study. First, the $R^{2}$ value measures how well the model captures the variability of the data. Based upon the authors' experience an $R^{2}$ value of 0.99 or better is achievable using POST data produced by the multiPOST tool. For the example problem, the $R^{2}$ of the fit for injected mass was 0.9981 .

Another goodness of fit test involves the residual by predicted plot. This plot shows the value predicted by the RSE plotted versus the error of each point. The desired pattern in the residual by predicted plot is a "shotgun spread", which can be seen in Figure 4 below. Curved patterns or clustering on the residual by predicted plot typically indicate higher order terms are needed. Note that the residual and predicted values have been normalized prior to plotting.

From the residual by predicted plot a very quick estimate for the percent error can be derived. Due to the normalization of the data in Figure 4 it is especially easy to derive as the y-axis essentially shows the percent error. Figure 5 shows the distribution of the percent error of the injected mass model. As seen in the figure the maximum absolute value of the error is 2.79 percent. The distribution shows that approximately $95 \%$ of the cases fall within $1 \%$ error. The standard deviation and mean of the percent error distribution are typically used to accept or reject the model. A standard deviation of less than 1 is desired, with a mean of nearly zero. In this case, the model is well below a standard deviation of 1 and has a mean of nearly 0 . 


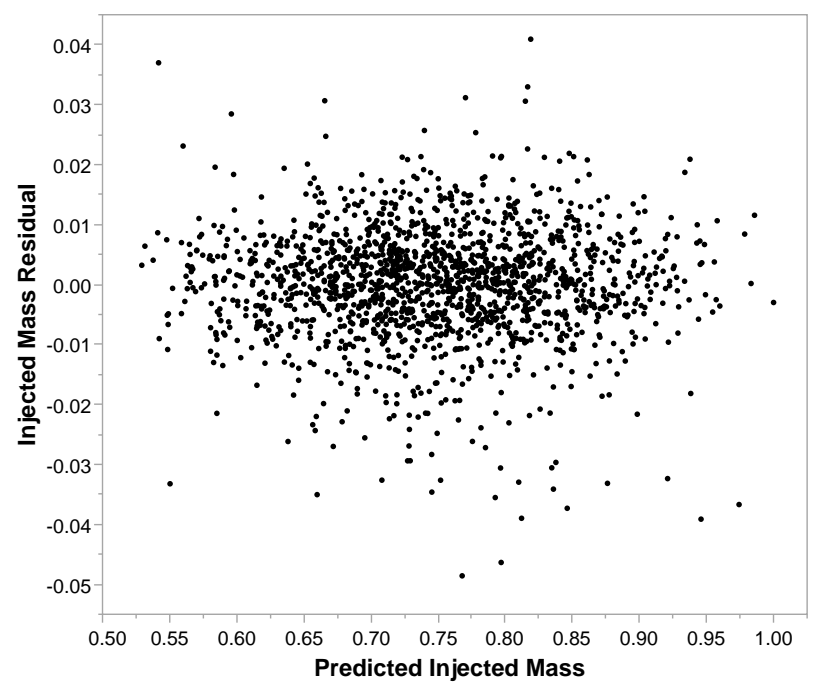

Figure 4: Residual by predicted plot for injected mass fit
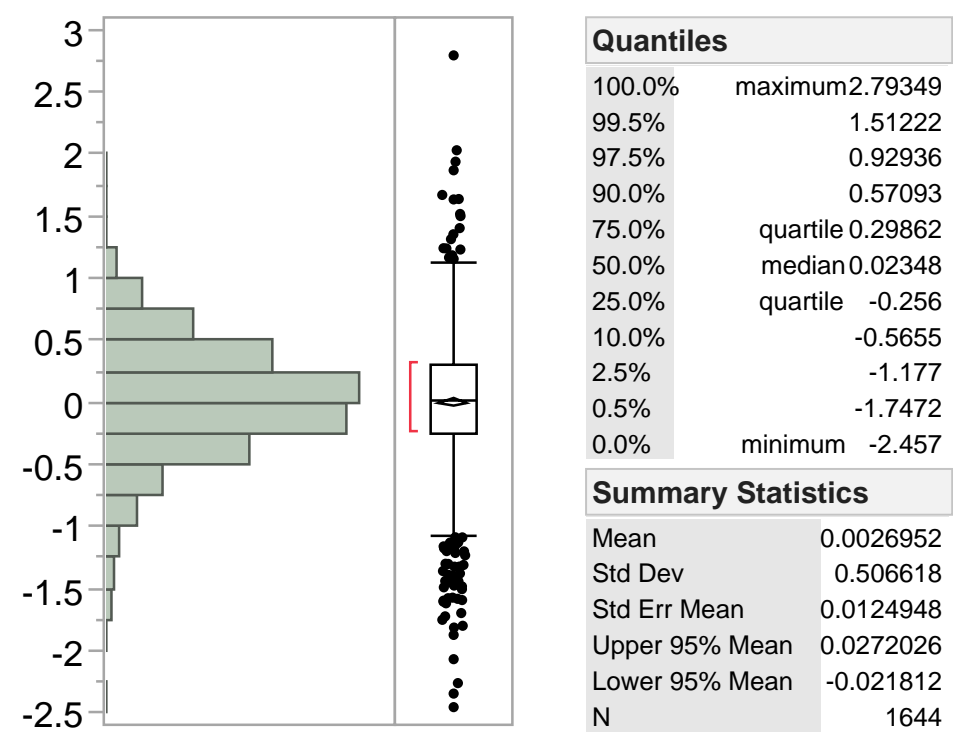

Figure 5: Percent error distribution for injected mass fit

During goodness of fit testing, Figure 4 above typically represents the model fit error, which is the error between the model prediction and the output of the points used to fit the model. Another percent error plot is produced for the model representation error, which is the error between the prediction and the output of points not used to fit the model. In this case the model representation error distribution is not necessary due to the method used to fit the model.

As discussed above, the stepwise personality was utilized in JMP to fit an RSE for injected mass. An option called $\mathrm{k}$-folds cross validation was implemented during the fit. This option splits the set of cases into $\mathrm{k}$ number of subsets. A model is then fit to the points in $\mathrm{k}-1$ subsets, while the last subset is held back for validation. This operation is performed for all combinations of the subsets. For example, using 3 folds would require 3 models be fit. These models would use the following subsets as fit data, A/B, B/C, A/C with the remaining set held back for validation. The implementation of $\mathrm{k}$-fold cross validation therefore rolls the model representation error goodness of fit test into the fitting of the model itself.

The goodness of fit tests were successfully implemented on the RSE for the example problem. The percent error of the response was deemed to be within acceptable tolerances for conceptual design. An illustration of the usefulness of the trajectory surrogate will be given below. 
Now that an RSE has been generated, it can be put to use. Figure 6 gives a JMP profiler illustration of the POST surrogate model for the example problem. In this figure, the POST surrogate inputs are listed in separate boxes along the $\mathrm{x}$-axis with the predicted injected mass on the $\mathrm{y}$-axis. These inputs are the burnout mass of the Core stage, exit area of the upper stage engines, gross mass of the upper stage, specific impulse of the upper stage engines, Thrust-to-Weight ratio of the upper stage, maximum axial loading in units of surface gravity g, and maximum dynamic pressure in units of psf, respectively. The curves in each box depict the predicted output over the range of settings for the specific input. Note that each curve is generated with all other inputs fixed.

The profiler very quickly provides the sensitivity of the injected mass to the various input parameters. As seen in the figure, the upper stage exit area, max acceleration, and max dynamic pressure have very little effect on the output. The upper stage thrust to weight shows the largest effect on the injected mass, decreasing drastically at the lower end of the input range.

In addition to providing sensitivities the profiler enables setup and execution of a Monte Carlo simulation using the surrogate model. This allows for the generation of very large data sets for further exploration of the design space. Such simulations can be used for technology evaluation, risk analysis, and inverse design. ${ }^{21,22}$

Figure 7 shows the Monte Carlo simulation setup in the JMP profiler window. The distributions on each of the inputs can be fully customized and the number of cases can be selected by the user. After running the simulation the expected injected mass given the input distributions is shown to the right of the original profiler. Note that the points can also be exported to a new JMP table for further investigation and plotting. The profiler examples give only a small glimpse at the analyses that can be performed after fitting a POST surrogate.

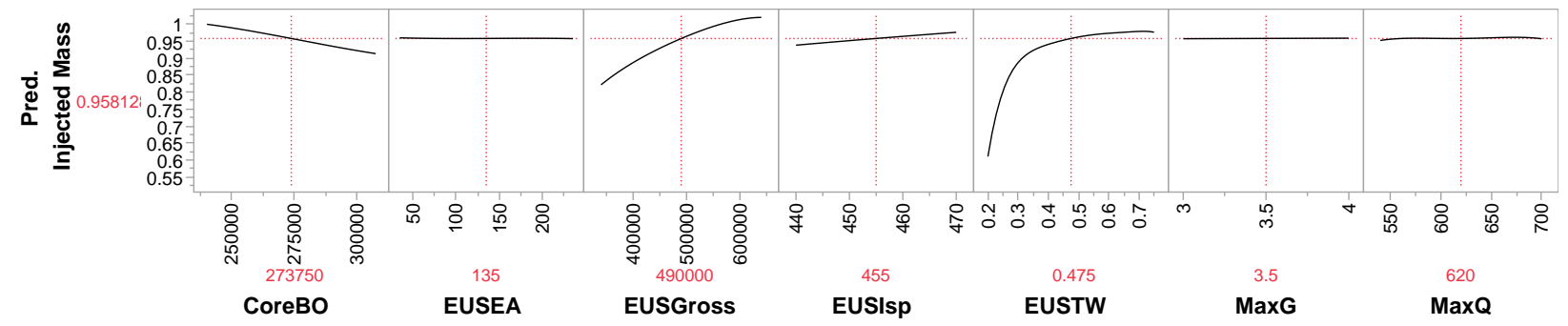

Figure 6: Prediction profiler for POST injected mass surrogate

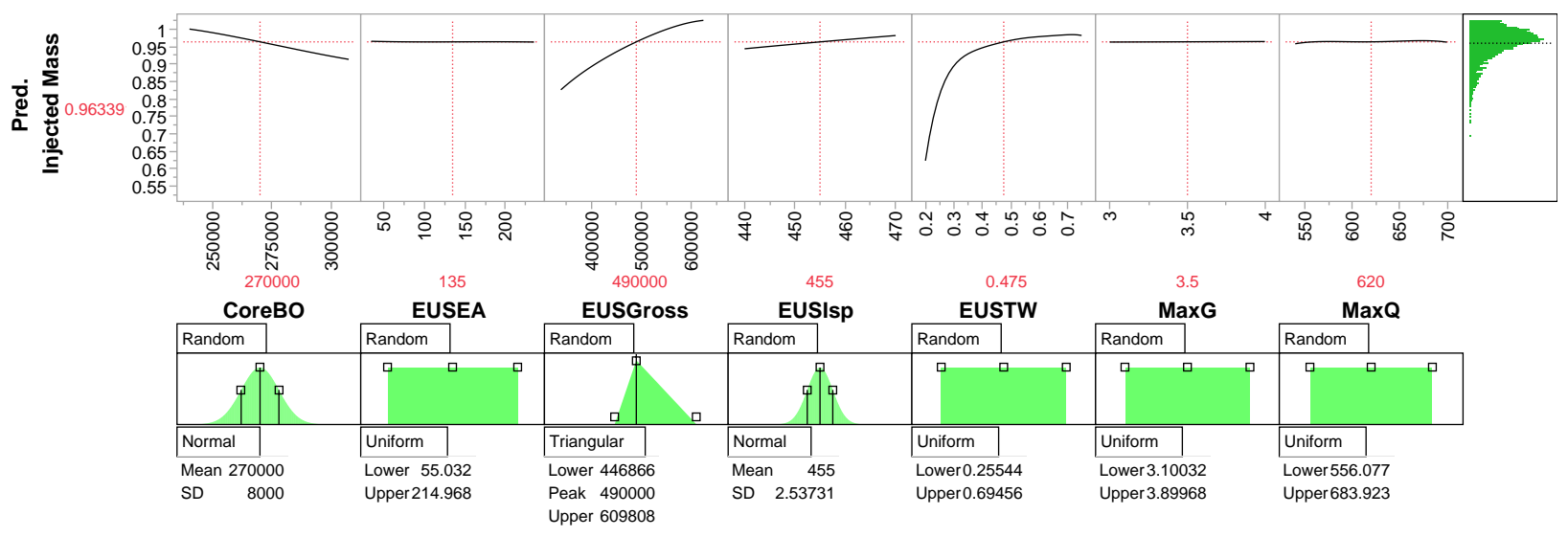

Figure 7: Prediction profiler with Monte Carlo simulation 


\section{Conclusion}

The primary goal of this paper was to develop a method for automating the execution of the POST trajectory optimization tool. Automating POST was motivated by the desire to generate a surrogate model for rapid trade space exploration, which requires large sets of data for fitting. In Section III a heuristics based approach was developed for automatically closing cases in POST. This method was demonstrated on an example problem in Section VI to illustrate its ability to successfully accomplish the initial goal and meet the method requirements defined in Section II.

The example problem utilized an SLS like vehicle with a parameterized upper stage. The multiPOST tool was implemented using the variables and ranges listed in Table 1. It successfully completed 31,000 out of 62,500 total repetitions defined by the DOE. Throughout the DOE execution, the multiPOST tool evaluated a large upper stage design space while successfully handling failed trajectories and POST program crashes. The example problem illustrated the time required for DOE completion. Using two computers, the multiPOST tool completed the DOE cases in approximately 48 hours. Including extra time for post processing this means, for a reasonable trade space, a POST surrogate can be completed in less than a week. This is well within the bounds of a typical conceptual design study, which meets the third method requirement.

The final requirement in Section II touched on validation and verification of the multiPOST tool. Applying $\mathrm{V} \& \mathrm{~V}$ is a very important step to ensure that an accurate surrogate model is produced. Without performing this step the surrogate model may grossly misrepresent the trade space. In section III heuristics were built into the multiPOST tool in order to capture expert knowledge. For every new trade study the heuristics can be adjusted to appropriately handle new vehicle configurations. In addition, the POST analyst must set up a parameterized input deck prior to DOE execution. These steps represent verification exercises that are built into the tool execution. Prior to running DOE cases the analyst is required to verify the successful operation of the input deck along with the heuristics.

Validation of the multiPOST tool execution can also be accomplished in multiple ways. First, the detailed input and output data for each case in the DOE can be saved as the tool executes. This allows the analyst to select any number of points from the DOE to validate via a comparison to the same case run by hand. Additional validation can be performed following the response surface fitting. This would involve a comparison between an analyst run case and the output of the surrogate model.

The multiPOST tool successfully meets the requirements stated in Section II. It allows for the generation of trajectory surrogate models for rapid design space exploration during conceptual design. Although the surrogate in Figure 7 provides a small number of variables to play with, it can capture more detailed trades by including other analysis tools. The Advanced Concepts Office at NASA Marshall Spaceflight Center is currently implementing the sizing and structural analysis tools, INTROS and LVA, in complement to POST. These sizing tools can capture the effects of parameters such as number of engines, engine mass, mixture ratio, and component materials on the burnout and gross masses in the POST surrogate. Linking surrogates of the sizing tools to the POST surrogate via core and upper stage masses can therefore support more detailed trades during conceptual design. Completion of these trades signifies an improvement in design knowledge that can be used for concept down selection, which ultimately contributes to the production of more affordable space launch vehicles. 


\section{References}

${ }^{1}$ Hertzfeld, H., Williamson, R., and Peter, N., "Launch Vehicles: An Economic Perspective," Tech. rep., The George Washington University Space Policy Institute, Washington, D.C., September 2005.

${ }^{2}$ Ryan, R. S. and Townsend, J. S., "Fundamentals and issues in launch vehicle design," Journal of Spacecraft and Rockets, Vol. 34, No. 2, 1997, pp. $192-198$.

${ }^{3}$ Department of Defense, Defense Aquisition Guidebook, September 2013. https://dag.dau.mil/Pages/Default.aspx.

${ }^{4}$ Blair, J., Ryan, R., Schutzenhofer, L., and Humphries, W., "Launch Vehicle Design Process: Characterization, Technical Integration, and Lessons Learned," Tech. rep., National Aeronautics and Space Administration, May 2001.

${ }^{5}$ Dulac, N. and Leveson, N., "Incorporating safety risk in early system architecture trade studies," Journal of Spacecraft and Rockets, Vol. 46, No. 2, 2009, pp. $430-437$.

${ }^{6}$ Ullah, R., Zhou, D.-Q., Zhou, P., Hussain, M., and Amjad Sohail, M., "An approach for space launch vehicle conceptual design and multi-attribute evaluation," Aerospace Science and Technology, 2011.

${ }^{7}$ Zwack, M., A Conceptual Reliability Growth Approach for Comparison of Launch Vehicle Architectures, Ph.D. thesis, Georgia Institute of Technology, Atlanta, GA, December 2014.

${ }^{8}$ Simpson, T., Booker, A., Ghosh, D., Giunta, A., Koch, P., and Yang, R., "Approximation Methods in Multidisciplinary Analysis and Optimization: A Panel Discussion," 9th AIAA/ISSMO Symposium on Multidisciplinary Analysis ES Optimization, Atlanta, GA, September 2002.

${ }^{9}$ Steffens, M., A Combined Global and Local Methodology for Launch Vehicle Trajectory Design-space Exploration and Optimization, Master's thesis, Georgia Institute of Technology, 2014.

${ }^{10}$ Waters, E., Garcia, J., Beers, B., Philips, A., Holt, J., and Threet, G., "NASA Advanced Concepts Office, Earth-To-Orbit Team Design Process and Tools," AIAA SPACE 2013 Conference $\mathcal{G}$ Exposition, 2013.

${ }^{11}$ Mulqueen, J., Maples, D., and Fabisinski, L., "Tailoring Systems Engineering Processes in a Conceptual Design Environment: A Case study at NASA Marshall Spaceflight Center's ACO," INCOSE International Symposium, Rome, Italy, July 2012.

${ }^{12}$ Ryan, T. P., Modern Experimental Design, Wiley Series in Probability and Statistics, 2007.

${ }^{13}$ Braun, R., Moore, A., and Kroo, I., "Use of the Collaborative Optimization Architecture for Launch Vehicle Design," 6th AIAA/USAF/NASA/ISSMO Symposium on Multidisciplinary Analysis and Optimization, 1996.

${ }^{14}$ Powell, R., Striepe, S., Desai, P., Braun, R., Brauer, G., Cornick, D., Olson, D., Peterson, F., and Stevenson, R., Program To Optimize Simulated Trajectories (POST) Vol II: Utilization Manual, 1997.

${ }^{15}$ Nelson, D., Qualitative and Quantitative Assessment of Optimal Trajectories by Implicit Simulation (OTIS) and Program to Optimize Simulated Trajectories (POST), Master's thesis, Georgia Institute of Technology, April 2001.

${ }^{16}$ Steffens, M. J., Edwards, S. J., and Mavris, D. N., "Capturing the Global Feasible Design Space for Launch Vehicle Ascent Trajectories," AIAA SciTech, Kissimmee, FL, January 2015.

${ }^{17}$ Hammond, D. P., "Parallelization of Program to Optimize Simulated Trajectories (POST3D)," Cr-2001-211250, NASA, November 2001.

${ }^{18}$ National Aeronautics and Space Administration, "NASA facts: Space Launch System," June 2012. http://www.nasa.gov/pdf/664158main_sls_fs_master.pdf.

${ }^{19}$ Aerojet Rocketdyne, "RS-25 Engine," Online, http://www.rocket.com/rs-25-engine.

${ }^{20}$ Alliant Techsystems Inc., "ATK Space Propulsion Products Catalog," September 2012. http://www.orbitalatk.com/flightsystems/propulsion-systems/launch-abort-motor/docs/orbital_atk_motor_catalog_(2012).pdf.

${ }^{21}$ Biltgen, P., Ender, T., and Mavris, D., "Development of a Collaborative Capability-Based Tradeoff Environment for Complex System Architectures," 44th AIA A Aerospace Sciences Meeting and Exhibit, Reno, Nevada, January 2006.

${ }^{22}$ Kirby, M., A Methodology for Technology Identification, Evaluation, and Selection in Conceptual and Preliminary Aircraft Design, Ph.D. thesis, Georgia Institute of Technology, May 2001. 